\title{
Perfluorophenylazide Functionalization of
}

\section{Electrospun Poly(para-dioxanone)}

\author{
Karola Luetzow ${ }^{1, \uparrow}$, Paul J. Hommes-Schattmannn ${ }^{1, \uparrow}$, Axel T. Neffe ${ }^{1,2}$, Bilal Ahmad ${ }^{3}$, Gareth R. \\ Williams $^{3}$, and Andreas Lendlein ${ }^{1,2, *}$
}

1: Institute of Biomaterial Science and Berlin-Brandenburg Centre for Regenerative Therapies, Helmholtz-Zentrum Geesthacht, 14513 Teltow, Germany.

2: Institute of Chemistry, University of Potsdam, Potsdam, Germany.

3: UCL School of Pharmacy, University College London, 29-39 Brunswick Square, London, WC1N 1AX, UK.

\footnotetext{
${ }^{\dagger}$ Both authors contributed equally to this work.

* Correspondence to: A. Lendlein, E-mail: andreas.lendlein@hzg.de
}

\begin{abstract}
:
Strategies to surface-functionalize scaffolds by covalent binding of biologically active compounds are of fundamental interest to control the interactions between scaffolds and biomolecules or cells. Poly(para-dioxanone) (PPDO) is a clinically established polymer that has shown potential as temporary implant, e.g. for the reconstruction of the inferior vena cava, as a nonwoven fiber mesh. PPDO lacks suitable chemical groups for covalent functionalization. Furthermore, PPDO is highly sensitive to hydrolysis, reflected by short in vivo half-life times and degradation during storage. Establishing a method for the covalent functionalization without degradation of this hydrolyzable polymer is therefore important to enable the surface tailoring for tissue engineering applications.
\end{abstract}


It was hypothesized that treatment of PPDO with a NHS-ester group bearing perfluorophenyl azide (PFPA) under UV-irradiation would allow efficient surface functionalization of the scaffold. XPS and ATR-FTIR investigation revealed the successful binding, while a GPC study showed that degradation did not occur under these conditions. Coupling of a rhodamine dye to the NHS-esters on the surface of a PFPA-functionalized scaffold via its amine-linker showed a homogenous staining of the PPDO in laser confocal microscopy. The PFPA method is therefore applicable even to the surface functionalization of hydrolytically labile polymers, and it was demonstrated that PFPA-chemistry may serve as a versatile tool for the (bio-)functionalization of PPDO scaffolds.

\section{Introduction}

Biomedical implants may be colonized by endogenous cells in vivo and ultimately be replaced by living tissue. A porosity of the material supporting the cell infiltration can e.g. be yielded by producing the scaffold through electrospinning a polymer ${ }^{1,2}$. In this respect, control over possible interactions between (electrospun) scaffolds and biomolecules or cells in general is of fundamental interest. Hence, various strategies to surface-functionalize scaffolds with biologically active compounds such as RGD-peptides, growth factors, or proteins have been studied to promote directed and non-directed cell adhesion ${ }^{3-6}$. Besides the possibility to display bioactive molecules on the scaffolds, a covalent chemical bond between the functionalizing entity and the scaffold is particularly useful to immobilize the active component on the scaffold surface. Polydioxanone (PPDO) is well-known as a suture in clinical use, however it has not achieved a breakthrough in tissue engineering despite studies on tendon, bone, and vascular tissue engineering. ${ }^{7-13}$ The chemical structure of PPDO lacks suitable functional groups for specific covalent coupling of biologically active substrates, which makes the surface functionalization by covalent reaction challenging. Thus, establishing a method for the covalent functionalization would offer a new prospective for PPDO in order to tailor its biointerface. 
PPDO is highly sensitive to degradation ${ }^{14-16}$, thus any functionalization reaction has to be carried out under non-aqueous conditions and the polymer integrity needs to be proven after the functionalization.

A versatile method to introduce specific motifs for covalent functionalization of various types of solid surfaces and nanoparticles, which has been applied to efficiently immobilize small molecules, proteins, enzymes, carbohydrates, and synthetic polymers, is perfluorophenylazide (PFPA) chemistry. Perfluorophenyl azides form highly reactive nitrene intermediates under UV-irradiation, and the latter readily undergo C-H insertion reactions with hydrocarbon-based materials. ${ }^{17}$ Only a few studies report on utilizing this methodology for surface functionalization of porous polymeric scaffolds, e.g. melt-blown poly(butylene terephthalate) (PBT) nonwoven fiber meshes ${ }^{18}$ or poly(4-methyl-1-pentene) (TPX) membranes. ${ }^{19}$ It was envisioned that the versatile PFPA-chemistry can be used by inserting the perfluorophenyl nitrene, which is generated by UV irradiation of the azide, into the aliphatic groups of PPDO. As the reaction can be performed in a non-aqueous environment, it was hypothesized that polymer hydrolysis can be avoided with this strategy. The reactive intermediates formed during the reaction may impair polymer integrity, however, such an occurrence has not yet been reported for other polyesters treated with PFPA. The azide needs to carry a moiety, which reacts readily under mild conditions in a subsequent reaction step, e.g. an $N$-hydroxysuccinimide (NHS) derived active ester that can be used for coupling of amine groups containing bioactive molecules to form an amide bond.

In this study, the functionalization of electrospun PPDO patches with NHS derived active ester moieties via PFPA-chemistry was investigated. Coupling was characterized by XPS and ATRFTIR spectroscopy. GPC analyses were conducted to show a potential impact on polymer integrity. For the subsequent coupling, a fluorescent dye was used to visualize the spatial distribution of the functionalization on the scaffold surface. 


\section{Experimental}

General information:

Methyl pentafluorobenzoate (1), sodium azide, 1-ethyl-3-(3-dimethylaminopropyl)carbodiimide hydrochloride, $N$-hydroxysuccinimide, and $\mathrm{N}$-(6-aminohexyl)rhodamine 6Gamide bis(trifluoroacetate) (5) were purchased from Sigma-Aldrich (Steinheim, Germany). Polydioxanone (PPDO; Resomer X206S, inherent viscosity of $1.5-2.2 \mathrm{dl} / \mathrm{g}[0.1 \mathrm{wt} . \%$ in hexafluoro-2-propanol (HFIP), $30^{\circ} \mathrm{C}$ ], residual monomer content: max. $0.5 \mathrm{wt} . \%$ ) was acquired in the form of white granules (Evonik Industries AG, Essen, Germany), and HFIP was obtained from Sigma-Aldrich (Gillingham, UK). Oligodioxanone (OPDO; $\mathrm{M}_{\mathrm{w}}=4470 \mathrm{~g} \cdot \mathrm{mol}^{-1} ; \mathrm{PDI}=$ 2.6) was synthesized according to literature. ${ }^{20}$ All other solvents were purchased from commercial suppliers in analytical grade and were used as received.

Synthesis of methyl 4-azidotetrafluorobenzoate (2):

The synthesis of $\mathbf{2}$ was performed under light exclusion (reaction vessels were covered with aluminum foil) according to literature procedures. ${ }^{21,22}$ Methyl pentafluorobenzoate $(\mathbf{1}, 2.0 \mathrm{~mL}$, $3.06 \mathrm{~g}, 13.6 \mathrm{mmol})$ was dissolved in a mixture of acetone $(20 \mathrm{~mL})$ and water $(10 \mathrm{~mL})$. Sodium azide $(1.15 \mathrm{~g}, 17.7 \mathrm{mmol})$ was added and the stirred mixture heated under reflux for $2 \mathrm{~h}$. The reaction mixture was diluted with water $(150 \mathrm{~mL})$ and was extracted with diethylether $(4 \times 60$ $\mathrm{mL}$ ). The combined organic layers were dried with magnesium sulfate and filtered. The solvent was removed under reduced pressure to give $2(3.22 \mathrm{~g}, 95 \%)$ as a colorless solid.

${ }^{1} \mathbf{H}$ NMR $\left(500 \mathrm{MHz}, \mathrm{CDCl}_{3}\right): \delta=3.97\left(\mathrm{~s}, 3 \mathrm{H}, \mathrm{OCH}_{3}\right) \mathrm{ppm}$.

${ }^{13} \mathbf{C}$ NMR (126 MHz, $\left.\mathrm{CDCl}_{3}\right): \delta=160.0\left(\mathrm{~s}, \mathrm{CO}_{2} \mathrm{CH}_{3}\right), 146.6-144.4(\mathrm{~m}, 2-\mathrm{F}, 6-\mathrm{F}), 141.8-139.5$ (m, 3-F, 4-F), 123.5 (tt, $\left.\mathrm{CN}_{3}\right), 107.8\left(\mathrm{t}, \mathrm{CCO}_{2} \mathrm{CH}_{3}\right), 53.4$ (q, $\left.\mathrm{OCH}_{3}\right)$ ppm. 
Synthesis of 4-azidotetrafluorobenzoic acid (3):

The synthesis of $\mathbf{3}$ was performed under light exclusion (reaction vessels were covered with aluminum foil) according to literature procedures. ${ }^{21,22} \mathbf{2}(3.22 \mathrm{~g},(12.93 \mathrm{mmol})$ was dissolved in methanol (14 mL) and aqueous sodium hydroxide solution (1.5 ml, $20 \mathrm{wt} . \%)$ was added. The reaction mixture was stirred for $4 \mathrm{~h}$ at room temperature. Water was added $(3 \mathrm{~mL})$ and $1 \mathrm{M}$ hydrochloric acid was added dropwise under stirring until the solution reached $\mathrm{pH}=1$. The solution was extracted with dichloromethane $(3 \times 60 \mathrm{~mL})$. The combined organic layers were dried with magnesium sulfate and filtered. The solvent was removed under reduced pressure to give $3(2.65 \mathrm{~g}, 87 \%)$ as a colorless solid.

${ }^{13}$ C NMR (126 MHz, DMSO-d6): $\delta=164.3$ (s, $\left.\mathrm{CO}_{2} \mathrm{H}\right), 144.3$ (d, 2-F, 6-F), 140.3 (d, 3-F, 4-F), $122.7\left(\mathrm{~s}, \mathrm{CN}_{3}\right), 108.4\left(\mathrm{~s}, \mathrm{CCO}_{2} \mathrm{H}\right) \mathrm{ppm}$.

Synthesis of $N$-succinimidyl 4-azidotetrafluorobenzoate (4):

The synthesis of $\mathbf{4}$ was performed under light exclusion (reaction vessels were covered with aluminum foil) according to a literature procedure. ${ }^{22}$ A mixture of 3 (1.25 g, $\left.5.74 \mathrm{mmol}\right)$, $\mathrm{EDC} \cdot \mathrm{HCl}(1.19 \mathrm{~g}, 6.21 \mathrm{mmol})$, and $\mathrm{N}$-hydroxysuccinimide $(0.72 \mathrm{~g}, 6.26 \mathrm{mmol})$ was dissolved in dichloromethane $(20 \mathrm{~mL})$ and reacted at $35^{\circ} \mathrm{C}$ for $24 \mathrm{~h}$ under stirring. Additional $\mathrm{EDC} \cdot \mathrm{HCl}$ $(0.62 \mathrm{~g}, 3.23 \mathrm{mmol})$ was added and stirring of the mixture was continued for $3 \mathrm{~h}$. The reaction mixture was diluted with water $(20 \mathrm{~mL})$ and extracted with dichloromethane $(3 \times 20 \mathrm{~mL})$. The combined organic layers were dried with magnesium sulfate and filtered, and the solvent was removed under reduced pressure to give crude $4(1.79 \mathrm{~g}, 94 \%)$ as a beige solid. The obtained product was recrystallized from 2-propanol $(0.2 \mathrm{~g} / \mathrm{mL})$ to give $\mathbf{4}$ as colorless needles.

${ }^{1} \mathbf{H}$ NMR $\left(500 \mathrm{MHz}, \mathrm{CDCl}_{3}\right): \delta=2.90\left(\mathrm{~s}, 4 \mathrm{H}, \mathrm{COCH}_{2}\right) \mathrm{ppm}$.

${ }^{13} \mathrm{C}$ NMR (126 MHz, $\left.\mathrm{CDCl}_{3}\right): \delta=168.4\left(\mathrm{~s}, \mathrm{COCH}_{2}\right), 155.3\left(\mathrm{t}, \mathrm{CO}_{2} \mathrm{~N}\right), 147.7-145.4(\mathrm{~m}, 2-\mathrm{F}, 6-$ F), 141.8-139.6 (m, 3-F, 5-F), $126.5\left(\mathrm{~s}, \mathrm{CN}_{3}\right), 102.1\left(\mathrm{t}, \mathrm{CCO}_{2} \mathrm{~N}\right), 25.8\left(\mathrm{t}, \mathrm{CH}_{2}\right) \mathrm{ppm}$. 
IR (ATR): $v=2995,2955(\mathrm{C}-\mathrm{H}), 2120\left(\mathrm{~N}_{3}\right), 1775,1735,1705,1640(\mathrm{C}=\mathrm{O}), 1480(\mathrm{C}=\mathrm{C}), 1410$, $1360,1250,1205,1135,1065,990 \mathrm{~cm}^{-1}$.

Preparation of electrospun PPDO patches:

PPDO (9\% w/v in HFIP) solutions were prepared by dissolving $0.9 \mathrm{~g}$ of PPDO in $10 \mathrm{~mL}$ of HFIP. The mixtures were stirred for 3 hours at room temperature to achieve complete dissolution of the polymer. The solutions were dispensed through a $20 \mathrm{G}$ stainless steel needle (ID $\sim 600 \mu \mathrm{m}$; Nordson EFD, Dunstable, UK) using a $10 \mathrm{~mL}$ polypropylene syringe mounted on a syringe pump (Cole-Parmer infusion pump EW-74900-05, Cole-Parmer, London, UK) at a flow rate of $1 \mathrm{~mL} / \mathrm{h}$. The needle was connected to a high-voltage DC power supply (HCP3535000, FuG Elektronik, Rosenheim, Germany). Aluminium foil was placed on top of the grounded collector that was used to collect the fibers. The distance from the needle to the collector was $12 \mathrm{~cm}$. Electrospinning was carried out at room temperature and relative humidity at $22{ }^{\circ} \mathrm{C}$ and $46 \%$.

PFPA-functionalization of the electrospun PPDO patches:

The electrospun patches were placed between a PTFE mask $(1.8 \times 4.7 \mathrm{~cm})$ and a glass plate and fixed with clips. Azide $4(0.2 \mathrm{~g})$ was dissolved in tetrahydrofuran $(2 \mathrm{~mL})$ and $1 \mathrm{~mL}$ of the solution casted onto the scaffolds. The solvent was allowed to evaporate under reduced pressure. After removal from the mask, the scaffolds were placed between two quartz plates, and irradiated for 5 min from each side (distance: $5 \mathrm{~cm}$; 5.4 A, $90 \%$ outpower) with a Exheimer Laser BlueLight ${ }^{\circledR C}$ Curing Module, BLC 30850 Hz (Hereaus, Hanau, Germany). The irradiated scaffolds were washed three times with tetrahydrofuran and dried under reduced pressure. 
PFPA-functionalization of OPDO:

$0.06 \mathrm{~g}$ OPDO was placed into a petridish (diameter $3 \mathrm{~cm}$ ). $0.09 \mathrm{~g}$ Azide 4 was dissolved in tetrahydrofuran $(1.5 \mathrm{~mL})$ and cast onto the OPDO powder. The solvent was allowed to evaporate under reduced pressure. The petridish was covered with a quartz plate and irradiated for 5 min (same conditions as for the PPDO patch), then the powder was mixed by stirring and irradiated a second time for $5 \mathrm{~min}$. The irradiated mixture was washed three times with $1.5 \mathrm{ml}$ tetrahydrofuran and dried under reduced pressure.

Coupling of rhodamine dye 5 to a PFPA-functionalized electrospun PPDO patch:

$N$-(6-Aminohexyl)rhodamine 6G-amide bis(trifluoroacetate) $(5,10 \mathrm{mg})$ was dissolved in tetrahydrofuran $(2 \mathrm{~mL})$. A small piece of a PFPA-functionalized elecrospun PPDO patch was immersed in the dye solution overnight at room temperature, followed by thorough washing with water to remove non-reacted physically adsorbed dye.

Instrumentation

Nuclear magnetic resonance spectroscopy (NMR):

NMR spectra were acquired on a DRX 500 Avance spectrometer (Bruker, Rheinstetten, Germany) at 298 K. Chemical shifts are reported relative to solvent residual peaks.

Attenuated total reflectance fourier-transformed infrared spectroscopy (ATR-FTIR):

ATR-FTIR spectra were recorded on a Nicolet 6700 FT-IR (Thermo Fisher Scientific, Waltham, MA, USA) spectrometer.

X-ray photoelectron spectroscopy (XPS):

The surface compositions of PFPA-functionalized PPDO patches and as-spun PPDO-patches were determined by XPS on a K-Alpha instrument (Thermo Scientific, East Grinstead, UK). 
The X-ray Source was a microfocused monochromatic Al-K $\alpha$ with an energy of $1486.6 \mathrm{eV}$. The surface compositions of the PFPA-functionalized OPDO powder and the pristine OPDO powder were analyzed by XPS on a Kratos Axis Ultra instrument (Kratos Analytica, Mancheseter, UK). A monochromatic Al K $\alpha$ beam source (1486.6 eV) was applied.

Typically three positions per sample were analyzed, with a spot size of $400 \times 800 \mu \mathrm{m}$, and an emission angle of $0^{\circ}$.

Scanning electron microscopy:

The morphological properties of electrospun PPDO patches were characterized using scanning electron microscopy (SEM; Quanta 200 FEG ESEM instrument, FEI, Hillsborough, OR, USA). Samples were sputtered with a gold coating (10 nm thickness) prior to SEM analysis. The images obtained were then analyzed using the ImageJ software and the fiber diameters (based on $>50$ measurements) were calculated. The jPOR plugin for ImageJ was additionally used to determine the porosity of the samples. In order to improve the reliability of the calculation, at least five SEM images were used to calculate the porosity for each sample.

Laser confocal microscopy:

The surface of the dye-functionalized PPDO patches and as-spun PPDO-patches were investigated on a confocal laser scanning microscope (LSM 780, Carl Zeiss, Jena, Germany) using a wavelength of $561 \mathrm{~nm}$.

Gel permeation chromatography:

Multidetector GPC measurements were performed at a solvent flow rate of $1 \mathrm{~mL} \cdot \mathrm{min}^{-1}$ at $35^{\circ} \mathrm{C}$ using chloroform as eluent and $0.2 \mathrm{wt} \%$ toluene as internal standard. The system was equipped with a precolumn, two $300 \mathrm{~mm}$ x $8.0 \mathrm{~mm}$ linear M columns (Polymer Standards Service GmbH 
(PSS), Mainz, Germany), an isocratic pump 2080, an automatic injector AS 2050 (both Jasco, Tokyo, Japan), a refractive index detector Shodex RI-101 (Showa Denko, München, Germany), and a dual detector T60A (Viscotek Corporation, Houston, USA). The SEC software WINGPC 6.2 (PSS) was used to determine the molecular weight distributions. Polystyrene samples were used for standard calibration.

\section{Results and Discussion}

The PPDO fiber meshes used in this study (Figure 1) were prepared by electrospinning PPDO solutions in HFIP $(9 \% \mathrm{w} / \mathrm{v})$. The obtained electrospun patches displayed a thickness of $85.6 \pm$ $50.7 \mu \mathrm{m}$, with a porosity of $27.8 \pm 2.0 \%$ (as determined from SEM images using the jPOR plugin in ImageJ) and an average fiber diameter of $0.91 \pm 0.42 \mu \mathrm{m}$ (Figure 1).

[Figure 1]

Many biologically active molecules, which are employed to promote cell adhesion to polymeric surfaces, such as peptides and proteins, contain amine groups. Amines readily react with $\mathrm{N}$ hydroxysuccinimide (NHS) derived active esters under mild conditions to form a covalent amide bond. A structurally simple PFPA derivative that has successfully been employed for introducing NHS active ester groups to polymeric surfaces, is perfluorophenyl azide 4. Its synthesis was performed following literature procedures as outlined in Scheme 1. In brief, methyl perfluorobenzoate (1) was reacted with sodium azide by nucleophilic aromatic substitution, providing methyl 4-azidoperfluorobenzoate $\mathbf{2}$. The methyl ester group of $\mathbf{2}$ was hydrolyzed under basic conditions to give $\mathbf{3}$, followed by carbodiimide promoted coupling of NHS to provide azide 4 with a yield of $78 \%$ over three steps.

[Scheme 1]

Electrospun PPDO patches were PFPA-functionalized by casting a THF solution of perfluorophenyl azide 4 onto the patches (as depicted in Scheme 2), followed by evaporation of the solvent and subsequent UV-irradiation using a UV excimer laser. The irradiated patch 
was thoroughly washed with THF to remove unreacted 4 . The patch remained stable during this functionalization procedure by judgement of the eye. To support this visual observation, it was decided to check the molar mass by GPC before and after functionalization (Figure 2) to exclude a possible degradation during the functionalization. Because the PPDO used for electrospinning was not soluble in solvents suitable for GPC, this investigation was carried out with a lower molecular weight OPDO, which is soluble in chloroform and was functionalized by a similar procedure. A lower molar mass of an analogue may, when end groups are participating in the degradation or are supporting e.g. solvent uptake, increase the susceptibility to degradation, but is unlikely to decrease it. ${ }^{23}$ It should be pointed out that not only the functionalization, but also the analysis of functionalization by XPS and ATR-FTIR of the electrospun patch and the model OPDO will be carried out in the same way to support that OPDO is a suitable model for PPDO. The analysis data will be discussed in the following at the appropriate place. The PFPA solution was casted on the OPDO powder, followed by evaporation of the THF and subsequent UVirradiation. Afterwards, the OPDO powder was thoroughly washed with THF, which should not dissolve the OPDO. GPC results revealed a trend for a slight increase of weight average molecular mass, and thus no degradation after functionalization $\left(\mathrm{M}_{\mathrm{w}}=4640 \mathrm{~g} \cdot \mathrm{mol}^{-1}\right.$; PDI $=$ 2.7). This is important, as OPDO/PPDO is very sensitive to hydrolysis. It should be noted that these values are according to standard calibration, as the broad molar mass distribution did not allow a universal calibration, and that the observed difference of $170 \mathrm{~g} \cdot \mathrm{mol}^{-1}$ compared to the $\mathrm{M}_{\mathrm{w}}$ of OPDO determined prior to the PFPA treatment is within the error of the method. The fact that the molar mass does not increase significantly, as one may expect when attaching PFPA molecules to the OPDO, can be rationalized when taking into account that only polymer chains on the surface of the OPDO powder particles can be possibly functionalized in this protocol and therefore the bulk of the polymer remains unchanged.

[Figure 2] 
In order to model the coupling of an amino group-containing substrate to the active ester moieties on the surface of the PFPA-functionalized patches, the fluorescent rhodamine dye $\mathbf{5}$ was coupled via its amine linker by treatment of a functionalized patch with a solution of $\mathbf{5}$ in THF, followed by washing to remove excess non-bound (physically adsorbed) 5. THF was chosen as the solvent, as it showed to retain the fiber structure of the patches (compare also Figure 3).

[Scheme 2]

To verify the successful coupling of 4 to the fiber surface, the PFPA-functionalized patches were analyzed by X-ray photoelectron spectroscopy (XPS) to determine their surface elemental composition. The elemental compositions of the surfaces of a pristine as-spun PPDO patch and a PFPA-treated patch (without coupling of rhodamine dye) are summarized in Table 1. As expected, the pristine electrospun patch contained no detectable amount of nitrogen (typical detection limits for XPS are \pm 0.1 atom $\%$ ) and only a very small amount of fluorine (about 0.3 atom $\%$, probably due to residual HFIP used for the spinning process) on the patch surface. The PFPA-treated patch, however, showed a nitrogen content of about 2.4 atom $\%$ and an increased amount of fluorine (about 1.9 atom\%). Although the accuracy of the obtained values have to be viewed critically (typically a relative error of at least $\pm 10 \%$ has to be assumed), the data clearly indicate the successful coupling of the perfluorophenyl azide 4 to the patch surface. The information from XPS typically reflects the composition of the surface up to about $10 \mathrm{~nm}$ depth. In analogy to the fiber, the OPDO powder was investigated by XPS. The XPS investigation of the pristine OPDO powder contained no detectable amount of nitrogen (as for the PPDO) nor of fluorine (in contrast to PPDO the OPDO had not been dissolved in HFIP). The carbon value of the PPDO $(63.2 \pm 0.2$ atom\%) is slightly higher than for OPDO (61.4 \pm 0.4 atom $\%)$, but also this could be due to traces of HFIP. The PFPA-functionalized OPDO powder revealed a nitrogen content of 0.3 atom $\%$ and a fluorine content of 0.5 atom $\%$. The values for nitrogen 
and fluorine of the PFPA-functionalized OPDO have a tendency to be slightly lower than of PFPA-PPDO, however the values are within the range of the relative error of this method.

\section{[Table 1]}

The success of the PFPA-functionalization protocol was further confirmed by ATR-FTIR spectroscopy. The IR spectra of perfluorophenyl azide 4, a pristine electrospun PPDO-patch, and a PFPA-treated patch are shown in Figure 4. The same investigation was carried out with the OPDO (pristine and PFPA functionalized powder) to ensure the compatibility of the PPDO and the OPDO, although for the OPDO spectra are not pictured completely because would lead to a loss of clarity. All spectra show small bands at wavenumbers between $3000 \mathrm{~cm}^{-1}$ and 2850 $\mathrm{cm}^{-1}$ from $\mathrm{C}-\mathrm{H}$ stretching vibrations. The spectrum of 4 shows a prominent signal at $2120 \mathrm{~cm}^{-1}$ corresponding to the asymmetric stretching vibration of the azide moiety, as well as several bands from the carbonyl vibrational bands between 1775 and $1640 \mathrm{~cm}^{-1}$, the latter one corresponding to the amide carbonyl bond of the NHS moiety. The spectra of the patches also show a typical carbonyl vibrational band from the ester groups of PPDO at $1735 \mathrm{~cm}^{-1}$. Comparison of the spectra of the pristine patch and the pristine powder to the one of the functionalized patch and the functionalized OPDO clearly reveals a small additional carbonyl vibrational band at $1650 \mathrm{~cm}^{-1}$ (see insert spectrum for the PPDO patch and for the OPDO powder) corresponding to the coupled NHS moiety. In case of the OPDO, this peak is slightly lower than for PPDO. From the IR spectra of the PPDO a coupling in the micromol range per $\mathrm{cm}^{2}$ can be roughly estimated. No differences were detected in the fingerprint region of the patch spectra. In addition to the XPS data, these spectra confirm the successful coupling of 4 to the PPDO-patch. Furthermore, both the OPDO and the PPDO show very similar results both in the XPS and in the FTIR investigations, even though the functionalization degree seems so be slightly lower for the OPDO. In summary, it can be concluded that OPDO is well suited as a model substance for PPDO. The absence of an azide vibrational band at $2120 \mathrm{~cm}^{-1}$ in the 
spectrum of the PFPA-functionalized patch shows that unreacted $\mathbf{4}$ was successfully removed from the patch by the washing procedure. The IR spectrum of the dye coupled PPDO patch showed no distinct difference in comparison to the spectrum of the pristine patch (data not shown), indicating that the concentration of the dye is too low to be detected by IR.

\section{[Figure 3]}

The coupling of the dye was therefore investigated by confocal laser microscopy. In addition to providing a visual proof for the dye coupling, this also gives information on the spatial distribution of the coupling that cannot be deduced from XPS and FTIR. Representative confocal laser microscopy images of the functionalized patches are given in Figure 3. As control experiments, a pristine PPDO patch (A) and a non-functionalized PPDO patch (B, no PFPAtreatment), that was treated with the dye in the same manner as the functionalized patch, are also depicted. The two images (C) and (D) of the dye-coupled patch show a red fluorescent staining of the electrospun PPDO fibres, indicating the successful covalent coupling of the dye. As expected, only the non-functionalized fibers of the PPDO patch (grey) are visible in (A), whereas a few red dots originating from physically adsorbed dye (red) are visible at high magnification in (B). Thus, it may be concluded that the PFPA-functionalization successfully provided NHS active ester moieties on the patch surface with a homogeneous distribution. It can further be judged that the fiber structure of the sample was retained.

This method allows the coupling of a variety of primary amine-containing bioactive molecules such as peptides and proteins: examples are the coupling of RGD-peptides ${ }^{18}$ allowing for nonselective adhesion of cells via their integrins, or the immobilization of enzymes ${ }^{24,25}$ and cell contact mediating proteins ${ }^{26}$ on PFPA-functionalized surfaces. Moreover, the NHS-ester moieties introduced by this method can be used to introduce also other functional groups, e.g. azides or alkynes, that have successfully been employed for carbohydrate immobilization via "click"- chemistry. ${ }^{22,27}$ 


\section{Conclusion}

In summary, this study demonstrates that the surface of electrospun PPDO can be functionalized efficiently via PFPA-chemistry to introduce NHS-ester moieties that are accessible for further functionalization by specific amine coupling without degrading the polymer. FTIR and XPS verified the presence of these NHS-moieties on the patch surface and confocal laser microscopy revealed a homogenous staining of the fibers after coupling of a fluorescent dye, indicating a broad and homogenous distribution of the dye. GPC data show that no degradation of the polymer occurs upon PFPA treatment and irradiation, which highlights the suitability of the method also for sensitive polymers. The method presented offers an attractive means for modulating the surface properties of electrospun polymeric scaffolds intended as biomedical devices or implants to control their biointerface.

\section{Acknowledgements}

This project has received funding from the European Union's Seventh Framework Programme for research, technological development and demonstration under grant agreement no 604049 and by Helmholtz Association through programme-oriented funding. X-ray photoelectron spectra for PPDO were obtained at the National EPSRC XPS Users' Service (NEXUS) at Newcastle University, an EPSRC Mid-Range Facility. The authors further thank David McCarthy for the provision of SEM images, Monique Hannemann for the synthesis of OPDO, Dr. Maria Balk for the GPC measurements, and Dr. Thomas Weigel for the X-ray photoelectron spectra of OPDO.

\section{References}

1. Jun I, Han HS, Edwards JR, Jeon H. Electrospun Fibrous Scaffolds for Tissue Engineering: Viewpoints on Architecture and Fabrication. Int J Mol Sci. 2018;19(3). 
2. Hong JK, Madihally SV. Next Generation of Electrosprayed Fibers for Tissue Regeneration. Tissue Eng Part B-Re. 2011;17(2):125-142.

3. Tallawi M, Rosellini E, Barbani N, et al. Strategies for the chemical and biological functionalization of scaffolds for cardiac tissue engineering: a review. $J R$ Soc Interface. 2015;12(108):20150254.

4. Krutty JD, Schmitt SK, Gopalan P, Murphy WL. Surface functionalization and dynamics of polymeric cell culture substrates. Curr Opin Biotech. 2016;40:164-169.

5. Kubo T, Wang X, Tong $Q$, Yan MD. Polymer-Based Photocoupling Agent for the Efficient Immobilization of Nanomaterials and Small Molecules. Langmuir. 2011;27(15):9372-9378.

6. Zhu JM. Bioactive modification of poly(ethylene glycol) hydrogels for tissue engineering. Biomaterials. 2010;31(17):4639-4656.

7. Boland ED, Coleman BD, Barnes CP, Simpson DG, Wnek GE, Bowlin GL. Electrospinning polydioxanone for biomedical applications. Acta Biomaterialia. 2005;1(1):115-123.

8. Goonoo N, Jeetah R, Bhaw-Luximon A, Jhurry D. Polydioxanone-based bio-materials for tissue engineering and drug/gene delivery applications. European Journal of Pharmaceutics and Biopharmaceutics. 2015;97(Part B):371-391.

9. Rowland $\mathrm{DCL}$, Aquilina $\mathrm{T}$, Klein $\mathrm{A}$, et al. A comparative evaluation of the effect of polymer chemistry and fiber orientation on mesenchymal stem cell differentiation. Journal of Biomedical Materials Research Part A. 2016;104(11):2843-2853.

10. Bottino MC, Yassen GH, Platt JA, et al. A novel three-dimensional scaffold for regenerative endodontics: materials and biological characterizations. Journal of Tissue Engineering and Regenerative Medicine. 2015;9(11):E116-E123.

11. Hakimi O, Mouthuy PA, Zargar N, Lostis E, Morrey M, Carr A. A layered electrospun and woven surgical scaffold to enhance endogenous tendon repair. Acta Biomaterialia. 2015;26(Supplement C):124-135.

12. Pontailler M, Illangakoon E, Williams GR, et al. Polymer-Based Reconstruction of the Inferior Vena Cava in Rat: Stem Cells or RGD Peptide? Tissue Engineering Part A. 2015;21(9-10):15521564.

13. Hommes-Schattmann PJ, Neffe AT, Ahmad B, et al. RGD constructs with physical anchor groups as polymer co-electrospinnable cell adhesives. Polymers for Advanced Technologies. 2017;28(10):1312-1317.

14. Bai W, Chen DL, Li Q, et al. In vitro hydrolytic degradation of poly(para-dioxanone) with high molecular weight. J Polym Res. 2009;16(5):471-480.

15. Liang BQ, Pang DL, Jin C, Li F, Wang YZ. Photo-oxidative degradation of poly(p-dioxanone) under ultraviolet light source. Polym Degrad Stabil. 2012;97(11):2162-2169.

16. Yang KK, Wang XL, Wang YZ, Huang HX. Effects of molecular weights of poly(p-dioxanone) on its thermal, rheological and mechanical properties and in vitro degradability. Mater Chem Phys. 2004;87(1):218-221.

17. Liu L-H, Yan M. Perfluorophenyl Azides: New Applications in Surface Functionalization and Nanomaterial Synthesis. Accounts of Chemical Research. 2010;43(11):1434-1443.

18. Gérard E, Bessy E, Hénard G, Ducoroy L, Verpoort T, Marchand-Brynaert J. Surface modification of poly(butylene terephthalate) nonwoven by photochemistry and biofunctionalization with peptides for blood filtration. Journal of Polymer Science Part A: Polymer Chemistry. 2011;49(23):5087-5099.

19. Möller L, Hess C, Paleček J, et al. Towards a biocompatible artificial lung: Covalent functionalization of poly(4-methylpent-1-ene) (TPX) with cRGD pentapeptide. Beilstein Journal of Organic Chemistry. 2013;9:270-277.

20. Zotzmann J, Ziegler HJ, Behl M, Zierke M, Radke W, Lendlein A. Upscaling the synthesis of biodegradable multiblock copolymers capable of a shape-memory effect. J Mater Sci-Mater M. 2011;22(10):2147-2154. 
21. Keana JFW, Cai SX. New reagents for photoaffinity labeling: synthesis and photolysis of functionalized perfluorophenyl azides. The Journal of Organic Chemistry. 1990;55(11):36403647.

22. Norberg O, Deng L, Yan M, Ramström O. Photo-Click Immobilization of Carbohydrates on Polymeric Surfaces-A Quick Method to Functionalize Surfaces for Biomolecular Recognition Studies. Bioconjugate Chemistry. 2009;20(12):2364-2370.

23. Hofmann D, Entrialgo-Castano M, Kratz K, Lendlein A. Knowledge-Based Approach towards Hydrolytic Degradation of Polymer-Based Biomaterials. Adv Mater. 2009;21(32-33):32373245.

24. Yan M, Cai SX, Wybourne MN, Keana JFW. Photochemical functionalization of polymer surfaces and the production of biomolecule-carrying micrometer-scale structures by deepUV lithography using 4-substituted perfluorophenyl azides. Journal of the American Chemical Society. 1993;115(2):814-816.

25. Yan M, Cai SX, Wybourne MN, Keana JFW. N-Hydroxysuccinimide Ester Functionalized Perfluorophenyl Azides as Novel Photoactive Heterobifunctional Crosslinking Reagents. The Covalent Immobilization of Biomolecules to Polymer Surfaces. Bioconjugate Chemistry. 1994;5(2):151-157.

26. Van Vlierberghe S, Vanderleyden E, Boterberg V, Dubruel P. Gelatin Functionalization of Biomaterial Surfaces: Strategies for Immobilization and Visualization. Polymers. 2011;3(1):114.

27. Li L, Li J, Kulkarni A, Liu S. Polyurethane (PU)-derived photoactive and copper-free clickable surface based on perfluorophenyl azide (PFPA) chemistry. Journal of Materials Chemistry $B$. 2013;1(4):571-582. 


\section{Tables}

Table 1. Elemental surface composition of electrospun PPDO, OPDO powder, and electrospun PPDO and ODPO powder both functionalized with PFPA, determined by XPS (given as mean values of three measurements and standard deviation).

\begin{tabular}{lcccc}
\hline Sample & $\begin{array}{c}\text { Surface } \\
\text { C (at.\%) }\end{array}$ & $\begin{array}{c}\text { Surface } \\
\text { N (at.\%) }\end{array}$ & $\begin{array}{c}\text { Surface } \\
\text { O (at.\%) }\end{array}$ & $\begin{array}{c}\text { Surface } \\
\text { F(at.\%) }\end{array}$ \\
\hline Pristine PPDO patch & $63.2 \pm 0.2$ & 0.0 & $36.0 \pm 0.4$ & $0.3 \pm 0.2$ \\
Pristine OPDO powder & $61.4 \pm 0.4$ & 0.0 & $38.6 \pm 0.4$ & 0 \\
PFPA-functionalized PPDO & $63.6 \pm 0.3$ & $2.4 \pm 0.4$ & $32.0 \pm 0.5$ & $1.9 \pm 0.1$ \\
PFPA-functionalized OPDO & $61.0 \pm 0.5$ & $0.3 \pm 0.1$ & $38.2 \pm 0.4$ & $0.5 \pm 0.1$ \\
\hline
\end{tabular}




\section{Figure legends}

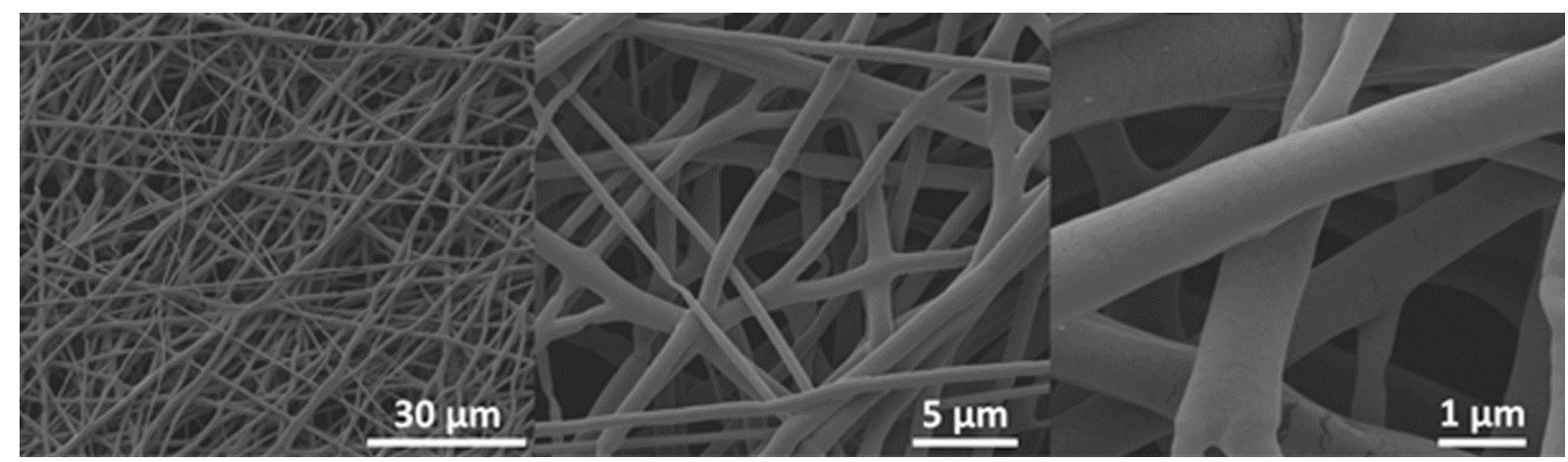

Figure 1. Electron microscopy pictures of an electrospun PPDO patch.

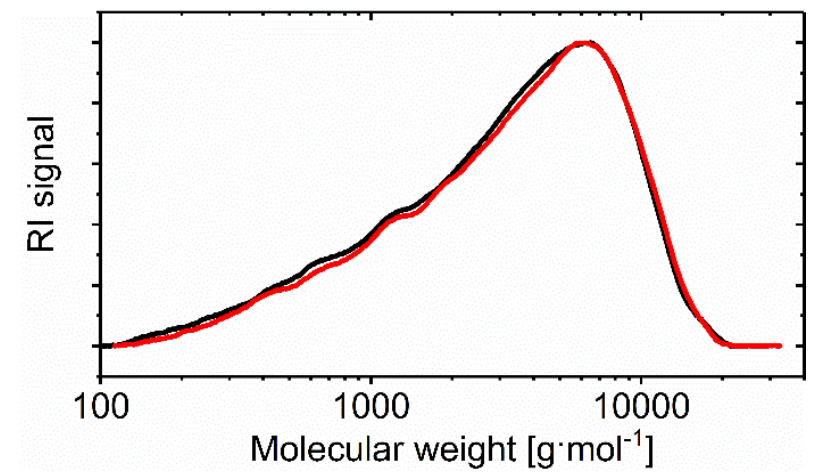

Figure 2. GPC chromatogram of OPDO before (black) and after (red) treatment with PFPA and irradiation. The $\mathrm{x}$-axis is scaled according to standard calibration. 

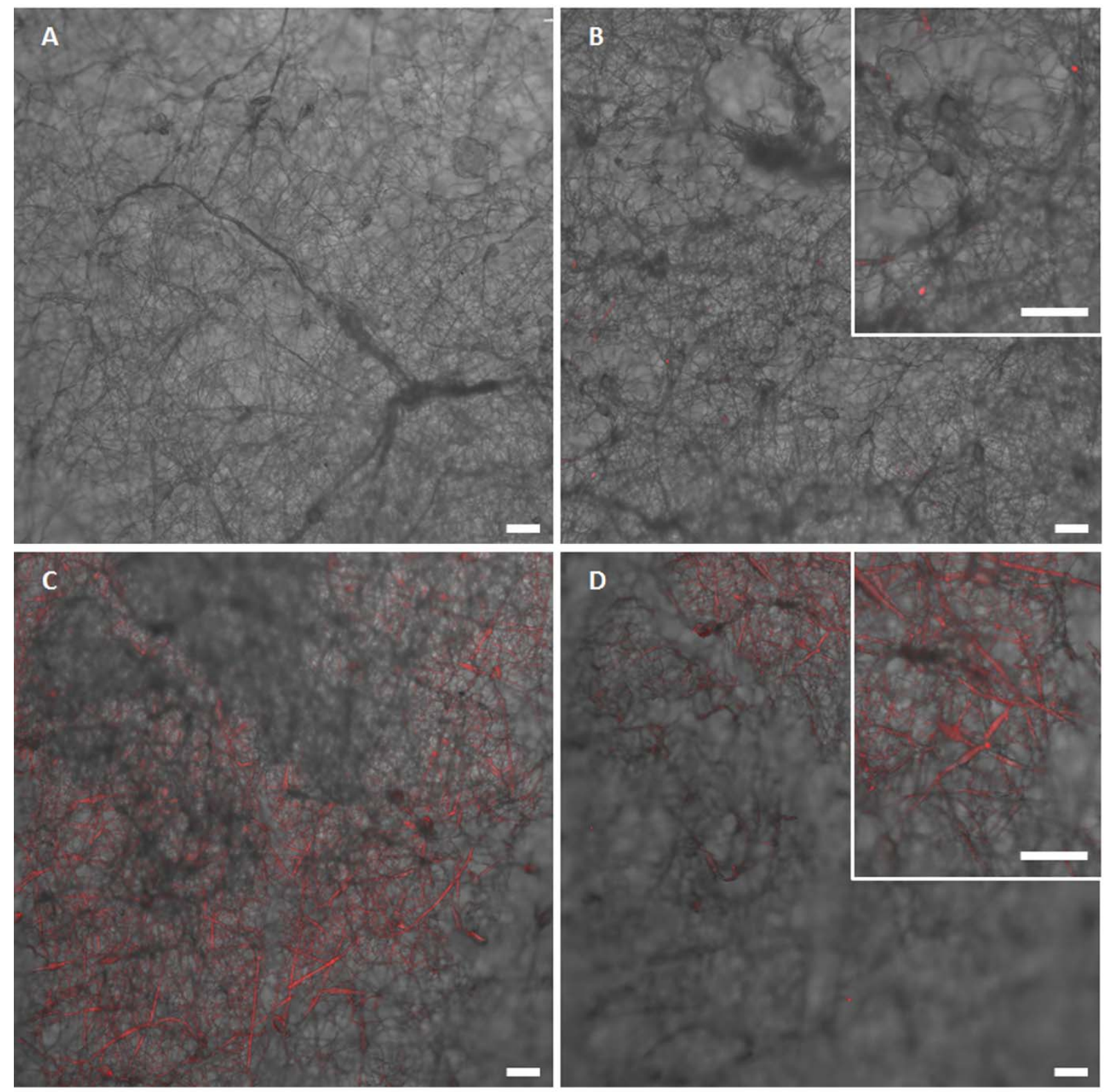

Figure 3. Confocal laser scanning microscope images of electrospun PPDO patches: (A) pristine (non-functionalized), (B) with physically adsorbed rhodamine dye, and (C+D) after PFPA-functionalization and coupling of rhodamine dye (images show the same sample at the same position with different focused levels). The scale bar represents $50 \mu \mathrm{m}$. (For interpretation, the reader is referred to the colored figure in the web version of the article.) 


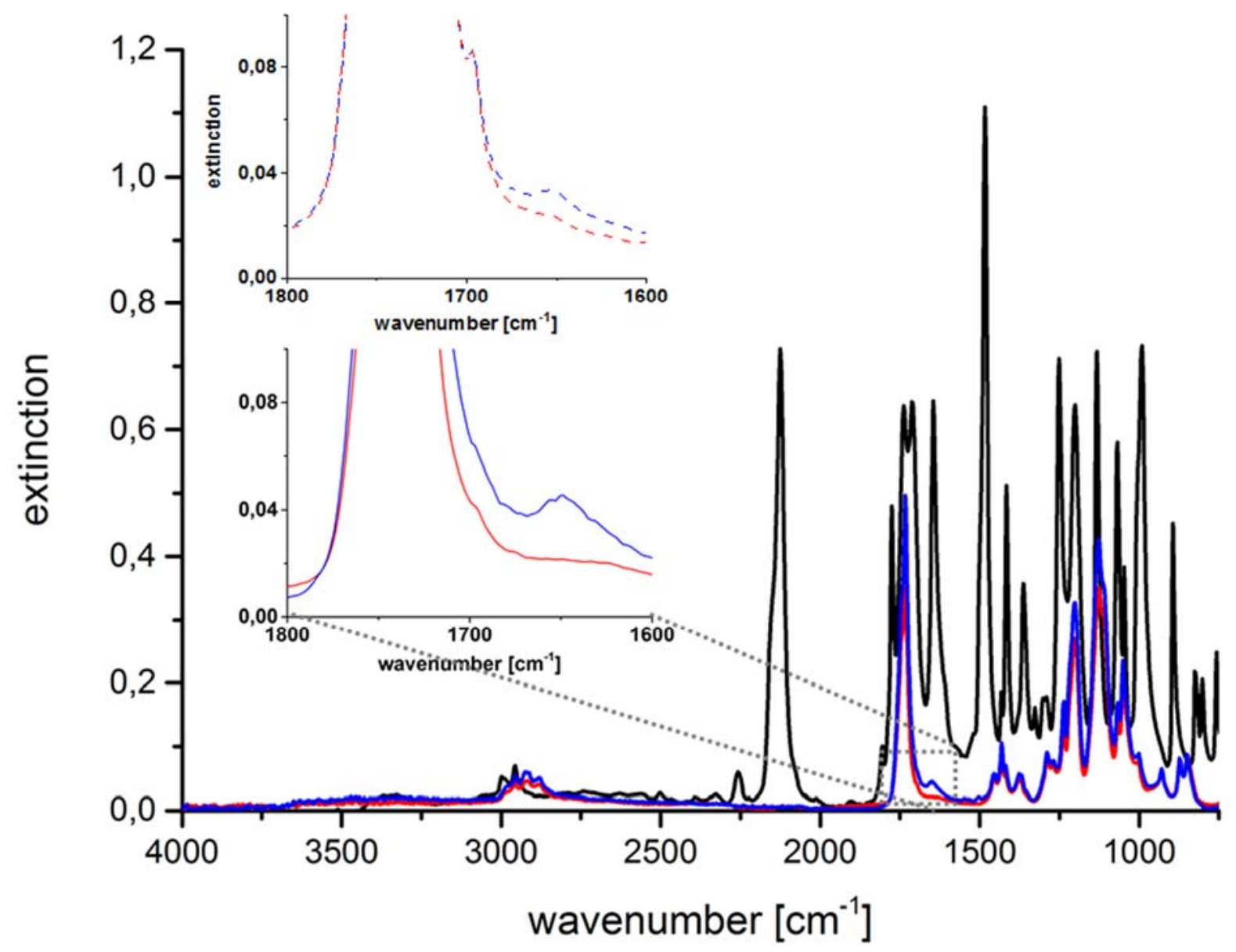

Figure 4. ATR-FTIR spectra of perfluorophenyl azide 4 (black graph), a pristine electrospun PPDO patch (red graph), OPDO (dashed red graph), a PFPA-functionalized PPDO-patch (blue graph), and a PFPA-OPDO (dashed blue graph). For better visualization only an excerpt (1800 $-1600 \mathrm{~cm}^{-1}$ ) is pictured for OPDO (For interpretation of the references to color in this figure legend, the reader is referred to the web version of the article.)

\section{Scheme legends}

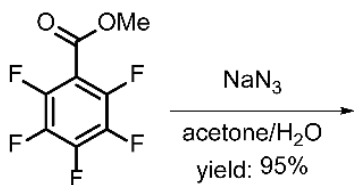

1

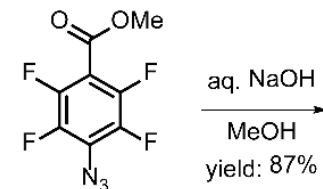

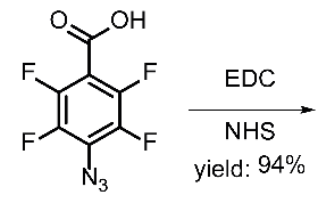

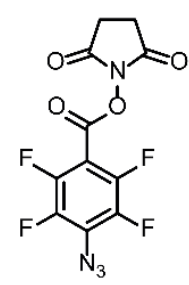

4

Scheme 1. Synthesis of perfluorophenylazide 4, performed following literature procedures. $^{21,22}$ 


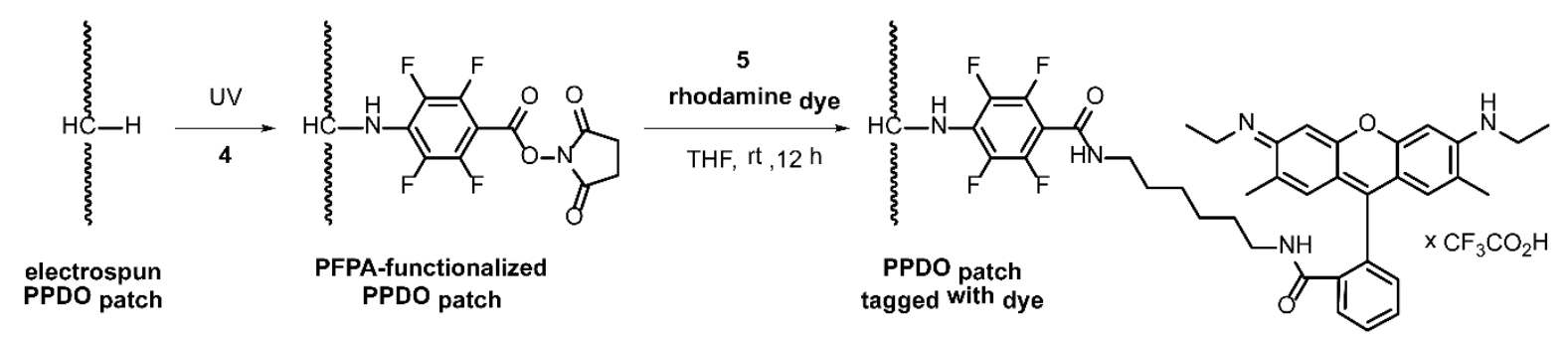

Scheme 2. PFPA functionalization of electrospun PPDO and coupling of a fluorescent rhodamine dye $\mathbf{5}$. 